\title{
TITLE:
}

\section{EQUATIONS GOVERNING CONVECTION IN EARTH'S CORE AND THE GEODYNAMO}

AUTHOR(S):

ROBERTS, PAUL H.

\section{CITATION:}

ROBERTS, PAUL H.. EQUATIONS GOVERNING CONVECTION IN EARTH'S CORE AND THE GEODYNAMO. 数理解析研究所講究録 1996, 970: 92-104

ISSUE DATE:

1996-10

URL:

http://hdl.handle.net/2433/60678

RIGHT: 


\title{
EQUATIONS GOVERNING CONVECTION IN EARTH'S CORE AND THE GEODYNAMO
}

\author{
PAUL H. ROBERTS \\ Department of Mathematics, and Institute of Geophysics \& Planetary Physics, \\ University of California, Los Angeles, California 90024 \\ Summary
}

A general strategy is presented for the study of convection in a turbulent fluid system such as Earth's core. This strategy is also applicable to systems much less complicated than Earth's core, which is a rapidly rotating magnetohydrodynamic body of metallic alloy that slowly evolves as it cools. These added complications must however be included when the Earth's magnetic field is explained by dynamo action in the core.

\section{Development of a Strategy}

This paper is a distillation of a paper with the same title by Braginsky \& Roberts (1995), which will be referred to as "BR", and of a recent paper describing a geodynamo simulation (Glatzmaier \& Roberts, 1996b). The latter, which will be referred to as "GR2", put the BR theory to work. Related simulations ("GR1" \& "GR1A") are described in Glatzmaier \& Roberts (1995a, 1995b for simulation GR1; 1996a for simulation GR1A). Figure 1 shows a rough sketch of Earth's interior, the system considered in this paper.

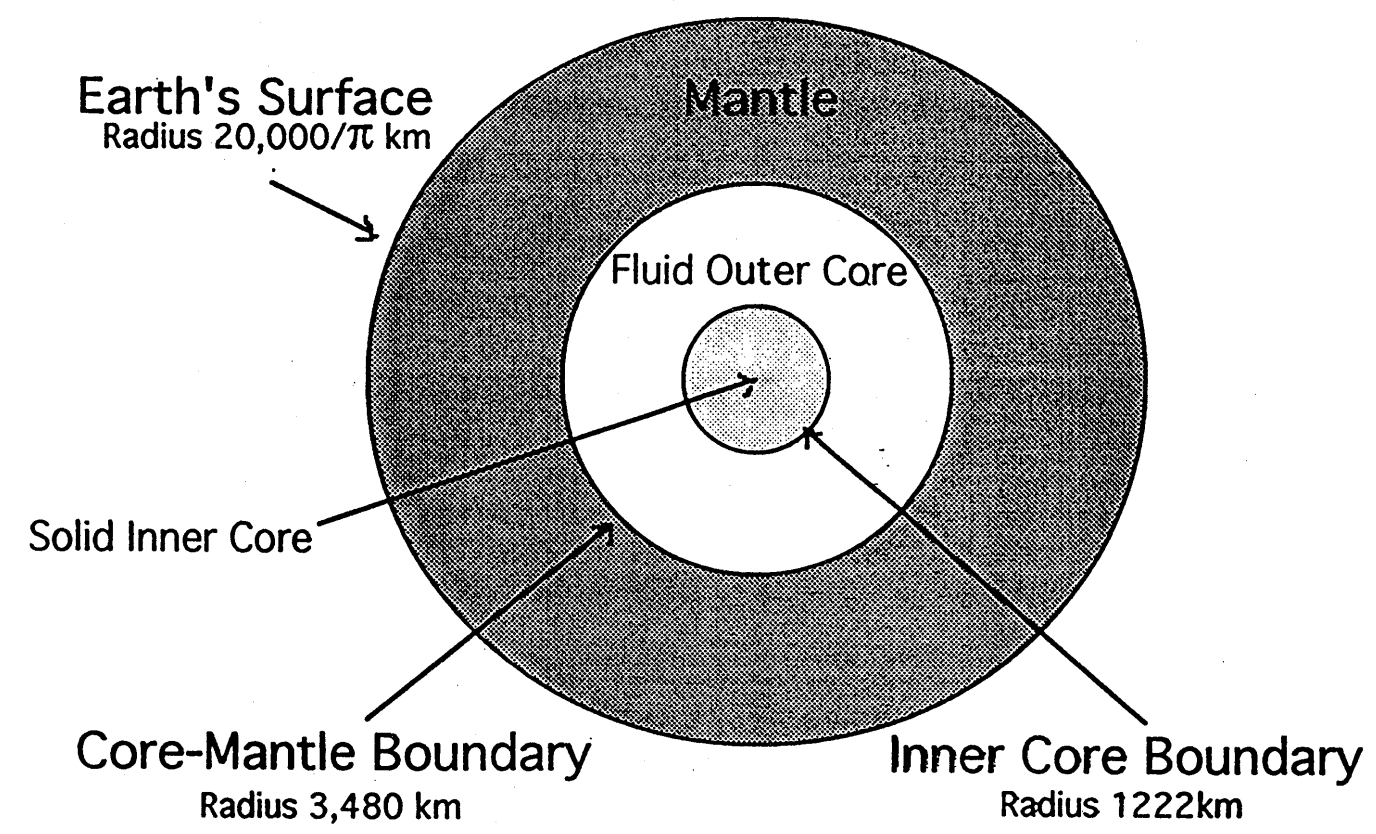

Fig. 1: A sketch of Earth's interior

GR1 \& GR1A are Boussinesq simulations that ignore the variation of density, $\rho$, in Earth's core due to the compression by the overlying material; simulation GR2 is an 
anelastic model which includes that variation, the assumed structure of Earth's interior being guided by the PREM model of Dziewonski \& Anderson (1981). The variation of core structure with depth is described by a parameter $\epsilon_{a}$, which is essentially the difference in $\rho$ at the inner core boundary ("ICB") and the core-mantle boundary ("CMB") divided by the mean density, and is of order 0.1. It is the smallness of this parameter that makes it reasonable to represent the core by a Boussinesq model, though doubtless the anelastic model is more satisfactory.

Convection in the core is so vigorous that all extensive quantities, such as the specific entropy, $S$, are very well mixed. To measure this effect, we may introduce a dimensionless parameter, $\epsilon_{c}=S_{c} / S_{a}$, where $S_{c}=S-S_{a}$ is the departure of $S$ from its value, $S_{a}$, in the well-mixed state; it is found that $\epsilon_{c} \sim 10^{-8}$. As is usual in convection theory, we describe convection in two steps: first we select a convenient reference state, and second we study departures from that reference state associated with convection. It is clear that a convenient reference state is the well-mixed state, that has uniform entropy for anelastic models and uniform temperature for Boussinesq models. GR1 \& GR1A are driven steadily by a specified heat flux at the ICB; GR2 is driven by heat conducted out of the core into the mantle across the CMB. The reference state of GR2 therefore changes secularly on a geological timescale, $t_{a}$, as the Earth cools, whereas the reference states of GR1 \& GR1A are steady.

A further complication, in the case of the Earth, is that bouyancy is provided not only thermally but also chemically, by differences in composition. Though the core is "an uncertain mixture of all the elements", there seems to be little doubt that it is abundent in iron. There is general agreement that the fluid outer core ("FOC") is significantly less dense than iron would be at core pressures, and that alloying elements must be present. There is no consensus on what the alloying elements are, or even what the predominant light constituent is. From a theoretical point of view, the basic physics is satisfied by assuming the simplest possible case, in which there is only one alloying element its mass fraction being $\xi$. This too is well mixed by the convection, so that $\xi_{a}$ is almost uniform in space, though dependent on $t_{a}$. Seismological models of the Earth's interior show that the density of the solid inner core ("SIC") is closer to that of pure iron than is that of the FOC. There is a density jump across the ICB of about $0.55 \mathrm{gm} / \mathrm{cc}$, presumably because the value $\xi_{N}$ of $\xi$ at the top of the SIC is less than $\xi_{a}$. This is naturally the case if, as is now believed, the ICB is a freezing interface. A simple phase diagram which would lead to such a conclusion is shown in the upper part of Figure 2. A phase diagram usually shows the solidus and liquidus of a material as curves in $\xi T$-space. This is because they are generally discussed in contexts where variations in pressure, $p$, are unimportant. In reality the liquidus and solidus depend on the thermodynamic state of the material. They should therefore appear as surfaces in three-dimensions. The traditional diagrams showing them as curves are merely intersections of these surfaces with the appropriate constant $-p$ plane. In the context of the core it is appropriate to plot the solidus and liquidus in $\xi p S$-space and to project them onto the plane $S=S_{a}$. This is the way they are shown in Figure 2, where we focus attention on the left-hand solidus and liquidus only. On descending through the FOC from the CMB, we eventually encounter the ICB, where the pressure is such that mixed phases can co-exist. The fluid alloy freezes onto the SIC, releasing latent heat 


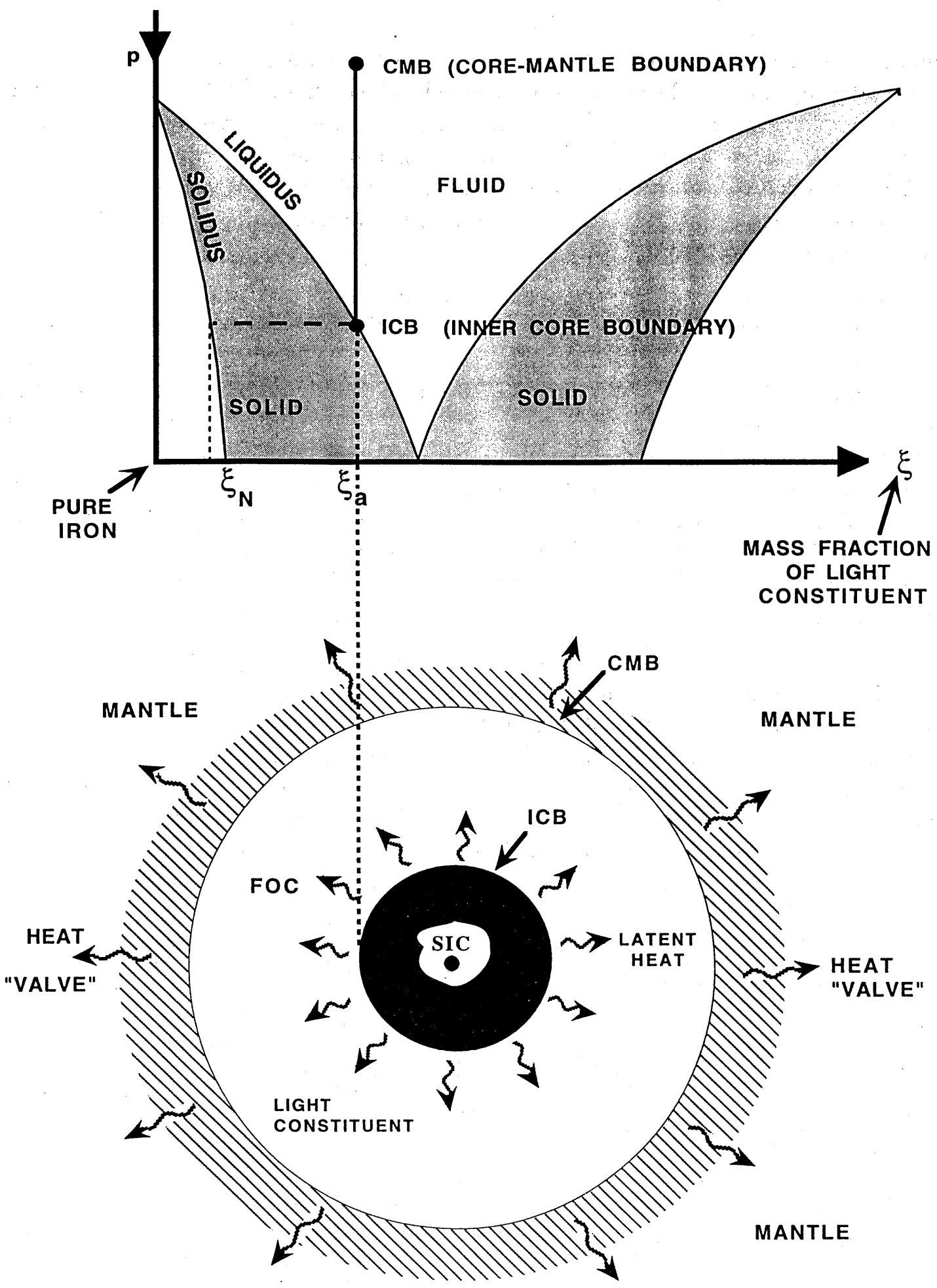

Fig. 2: Sketch of a phase diagram of core material (top), and its relationship to the core itself (bottom). 
and light constituent as it does so. These sources of buoyancy establish core convection that stirs the fluid, making $S_{a}$ and $\xi_{a}$ almost uniform, and generating the Earth's magnetic field by dynamo action. GR2 includes both these sources; GR1 \& 1A recognizes thermal buoyancy alone. It should perhaps be emphasized that, although it is necessary in GR2 to include both sources of buoyancy and to allow the reference state to evolve in time, these complications are not essential in other applications (and are not in fact included in GR1 \& GR1A). The theory we describe below, which contains a few novelties, therefore has wider applications. As in many other applications of convection theory, motions in Earth's core are on many length and time scales. That this must be the case is clear when we consider quantities such as the Péclet number, $\bar{P}=\overline{V L} / \kappa^{T}$, and the Schmidt number, $\bar{S}=\overline{V L} / \kappa^{\xi}$, of the macroscale defined by a characteristic macroscale velocity $\bar{V}$ and length $\bar{L}$ and by typical molecular diffusivities $\kappa^{T}$ and $\kappa^{\xi}$ of heat and composition. Taking $\bar{V}=10^{-4} \mathrm{~m} / \mathrm{s}$ and $\bar{L}=10^{6} \mathrm{~m}$, we find that $\bar{P} \sim 10^{7}$ and $\bar{S} \sim 10^{12}$. This shows that molecular transport of heat and composition is almost totally ineffective on the microscale. It is also true of the momentum transport as measured by the kinetic Reynolds number, $\bar{R}=\overline{V L} / \nu^{m} \sim 10^{5}$, where $\nu^{m}$ is the molecular kinematic viscosity. It is clear that, to transport heat, composition and momentum on the macroscale, the core has to develop small scale motions (turbulence).

The incorporation of turbulence into the theory is an unavoidable complication that has to be overcome in a physically consistent way. We adopt the simplest possible model: a local turbulence theory in which a field such as $S_{c}$ is divided into a macroscale part, $\bar{S}$, and a microscale part, $S^{\prime}$, e.g. ${ }^{1}$, We then develop expressions for the macroscale fluxes of entropy, composition and momentum at position $\mathbf{x}$ and time $t$ created by the turbulence. In a local theory, these depend on the macroscale properties of the system, but only at the same $\mathbf{x}$ and $t$. The evaluation of these fluxes requires separate and detailed study; see Appendix C of BR, the paper of Braginsky \& Meytlis (1990), and the discussion below.

The steps required to develop a workable theory of core convection and the geodynamo are now apparent; they are summarized in Table 0 and by the following supplementary remarks. Concerning step 2 of the table, the rearrangement of mass inherent in a cooling Earth can only be brought about by motions of order $V_{a} \sim 10^{-12} \mathrm{~m} / \mathrm{s} \sim \epsilon_{c} V_{c}$, which is so small that the momentum equation reduces to an expression of hydrostatic balance. Concerning step $3, \epsilon_{c}$ is so small that it is possible to linearize the thermodynamics about the well mixed state of uniform entropy and composition, though all other nonlinearities must be retained. We should also point out that seismic waves cross Earth's core in about 10 minutes. Not only is such a small time scale irrelevant to the phenomenon under study, but also to include it would require such short time steps in a numerical simulation that

1 The overbar on $\bar{S}$ denotes an average over turbulent ensembles and $S^{\prime}$ is the superimposed turbulent part of $S$. This notation is often used in mean field electrodynamics; see for example Krause \& Rädler (1980). It is different from that of BR who wrote $S_{c}=\left\langle S_{c}\right\rangle^{t}+S^{\dagger}$. They did this to avoid a possible ambiguity: an overbar is often used in geomagnetic theory to signify the axisymmetric part of a field, i.e., an average over longitude; a prime then signifies the asymmetric part of the field. 
Table 0: STRATEGY: Development of Basic Convection Equations

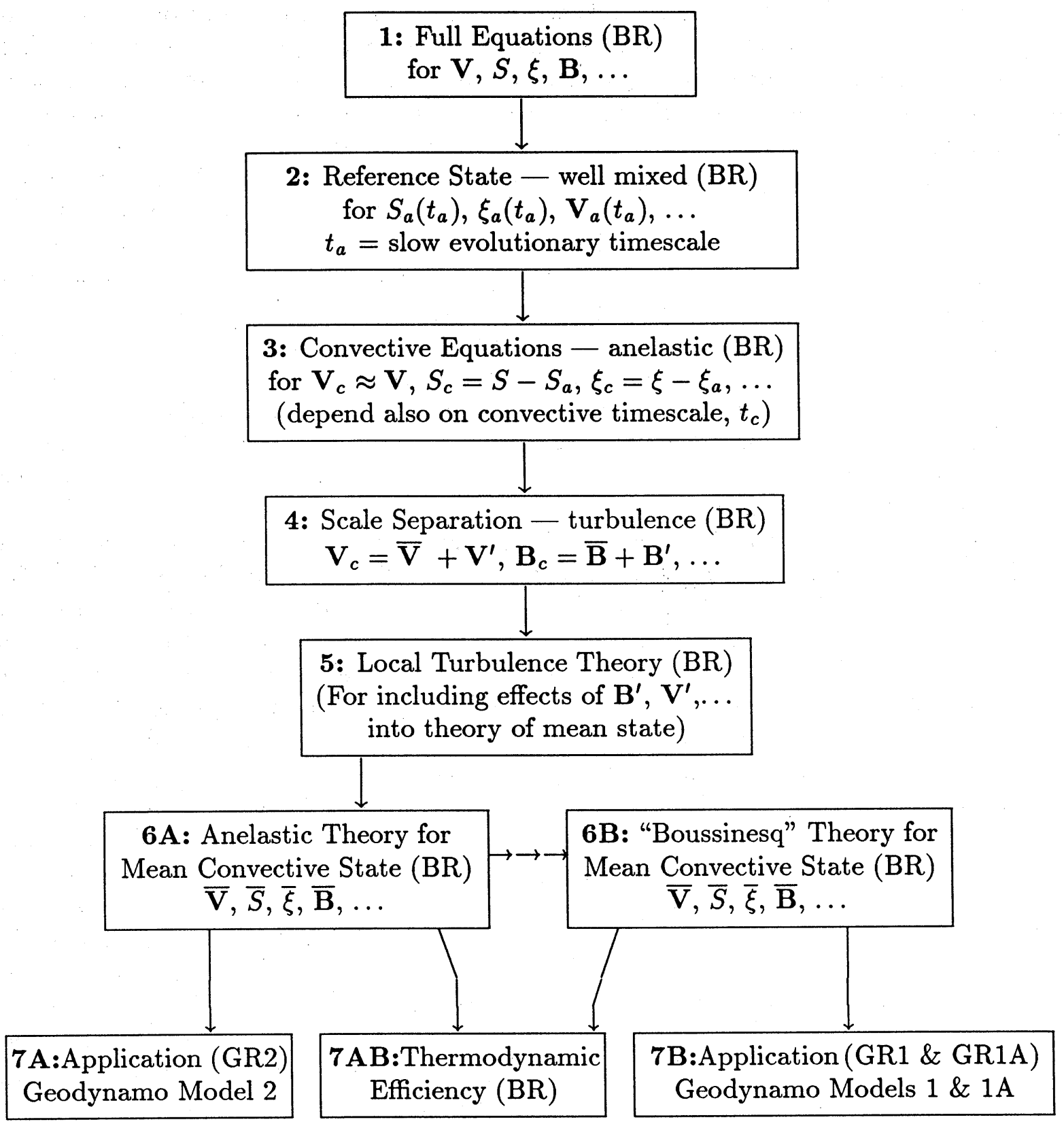

it would render it totally impractical ${ }^{2}$. We therefore adopt the anelastic approximation in which $\partial \rho_{c} / \partial t$ is neglected in the continuity equation. This "filters out" sound waves. Once

2 This statement, though true for Earth's core, is not universally applicable to all convective dyanamos. For example, Kageyama et al. (1995) consider convection in a highly compressible body of fluid. If their model were scaled to core conditions, sound would take decades to cross the core. Under such circumstances it would be incorrect to replace the continuity equation by the anelastic equation, and in fact they did not do so. 
the final anelastic equations have been derived (see step 6A), a reduction to a Boussinesqtype theory is possible (step $6 \mathrm{~B}$ ). This is described in $\S 8$ of $\mathrm{BR}$; an even simpler form of Boussinesq convection is used in the GR1 and GR1A simulations. The anelastic and Boussinesq-like theories have interesting thermodynamic implications concerning the thermodynamic efficiency of convective systems, regarded as heat engines (step 7AB). These are discussed in $\S 7$ of $B R$, but will not be described here. One further point should be mentioned. The gravity defining the reference state is the effective gravity, $\mathbf{g}_{\mathbf{e}}$, which differs from Newtonian gravity, $\mathbf{g}$, by the centrifugal acceleration due to Earth's rotation. The whole Earth (including the ICB and CMB) is therefore slightly flattened. The significance of this effect is measured by a further dimensionless parameter, $\epsilon_{\Omega}=\Omega^{2} \bar{L} / \bar{g} \sim 10^{-3}$, where $\Omega$ is the angular velocity of Earth and $\bar{g}$ is a characteristic gravitational acceleration. It is clear that the effect is not large, but to include it would add severe complications to the theory without any compensating enlightenment. We shall therefore later set $\epsilon_{\Omega} \equiv 0$, though we shall retain the Coriolis force. The steps leading from $6 \mathrm{~A}$ to $6 \mathrm{~B}$ may be found in $\mathrm{BR}$ and are not repeated in the present paper.

\section{The Strategy in Greater Detail}

The arguments that BR employed to complete the strategy set out in Table 0 are fully set out in BR and will therefore be described only briefly here. The basic equations (step 1 in Table 0 ) are summarized in Table 1, which also contains a short table of notation.

\section{Table 1: BASIC EQUATIONS}

\begin{tabular}{ll|}
$\rho d_{t} \mathbf{V}=-\nabla p+\rho \mathbf{g}_{\mathrm{e}}-2 \rho \Omega \times \mathbf{V}+\rho \mathbf{F}^{V}+\rho \mathbf{F}^{J}$, & $\partial_{t} \rho=-\nabla \cdot(\rho \mathbf{V})$ \\
$\rho d_{t} \xi=-\nabla \cdot \mathbf{I}^{\xi}$, & $\rho d_{t} S=-\nabla \cdot \mathbf{I}^{S}+\sigma^{S}$ \\
$\partial_{t} \mathbf{B}=\nabla \times(\mathbf{V} \times \mathbf{B})-\nabla \times(\eta \nabla \times \mathbf{B})$, & $\nabla \cdot \mathbf{B}=0$ \\
$\nabla^{2} U=4 \pi k_{N} \rho$ & \\
\hline
\end{tabular}

\section{Notation:}

$$
\begin{aligned}
& \mathbf{x}=\text { position; } \quad t=\text { time; } \quad \partial_{t}=\partial / \partial t=\text { Eulerian derivative } \\
& d_{t}=\partial_{t}+\mathbf{V} \cdot \boldsymbol{\nabla}=\text { Lagrangian (motional) derivative } \\
& \rho=\text { mass density } \quad \quad \mathbf{V}=\text { fluid velocity; } \quad \mathbf{B}=\text { magnetic field } \\
& T=\text { temperature; } \quad \mu=\text { chemical potential; } \quad p=\text { pressure; } \\
& S=\text { entropy } ; \quad \mathbf{I}^{S}=\text { entropy flux; } \quad \sigma^{S}=\text { volumetric entropy source } \\
& \xi=\text { mass fraction of light constituent; } \quad I^{\xi}=\text { flux of } \xi \\
& \mathbf{F}^{V}=\text { viscous force; } \quad \mathbf{F}^{J}=\mathbf{J} \times \mathbf{B}=\text { Lorentz force } \\
& \mathbf{g}_{\mathrm{e}}=\text { effective gravitational field }=\mathbf{g}-\boldsymbol{\Omega} \times(\boldsymbol{\Omega} \times \mathbf{x}) ; \quad k_{N}=\text { gravitational constant } \\
& \mathbf{g}=-\nabla U=\text { true gravitational field; } \quad U=\text { gravitational potential } \\
& \boldsymbol{\Omega}=\text { angular velocity of reference frame (mantle); } \quad \eta=\text { magnetic diffusivity }
\end{aligned}
$$


The reference state (step 2) is described in Table 2. Of particular interest here is the introduction of a generalized potential $\Pi$ such that $\Pi_{a}$ is, like $S_{a}$ and $\xi_{a}$, uniform in the reference state. In this table, $u_{S}$ is the speed of sound and $c_{p}$ is the specific heat at constant pressure. All such quantities are evaluated in the reference state.

\section{Table 2: REFERENCE STATE}

Well-mixed State in (approximate) Hydrostatic Equilibrium:

$$
\begin{array}{ll}
\nabla \xi_{a}=0, & \nabla S_{a}=\mathbf{0} \\
\rho_{a}^{-1} \nabla p_{a}=-\nabla U_{\mathrm{e} a} \equiv \mathbf{g}_{\mathrm{e} a}, & \nabla^{2} U_{\mathrm{e} a}=4 \pi k_{N} \rho_{a}-2 \Omega^{2} \\
\nabla \cdot\left(\rho_{a} \mathbf{V}_{a}\right)=-\dot{\rho}_{a}, & \mathbf{B}_{a}=0
\end{array}
$$

Variables are functions of $\mathbf{x}$ and of the slow time scale, $t_{a} ; \xi$ and $S$ are independent of $\mathbf{x}$. So is $\Pi=\varepsilon^{H}+U$, where $\varepsilon^{H}=$ enthalpy/unit mass. Hydrostatic equilibrium $\Rightarrow$

$$
\nabla \Pi_{a}=0
$$

Elementary Consequences:

$$
\rho_{a}=\rho\left(p_{a}, S_{a}, \xi_{a}\right), \quad T_{a}=T\left(p_{a}, S_{a}, \xi_{a}\right), \quad \mu_{a}=\mu\left(p_{a}, S_{a}, \xi_{a}\right)
$$

and

$$
\rho_{a}^{-1} \nabla \rho_{a}=\mathrm{g}_{\mathrm{e} a} / u_{S}^{2}, \quad \nabla \mu_{a}=\alpha^{\xi} \mathrm{g}_{\mathrm{e} a}, \quad \nabla T_{a}=\alpha^{S} \mathrm{~g}_{\mathrm{e} a}
$$

where

$$
\alpha^{\xi}=-\frac{1}{\rho}\left(\frac{\partial \rho}{\partial \xi}\right)_{p, S}, \quad \alpha^{S}=-\frac{1}{\rho}\left(\frac{\partial \rho}{\partial S}\right)_{p, \xi}=\frac{\alpha T}{c_{p}}
$$

More familiar form:

$$
T_{a}^{-1} \nabla T_{a}=\gamma \mathbf{g}_{\mathrm{e} a} / u_{S}^{2}, \quad \gamma=\alpha u_{S}^{2} / c_{p}=\text { the Grüneisen constant }
$$

The basic anelastic equations (step 3) are set out in Table 3, and a remarkable simplification discovered by BR is briefly set out. This consists of a new way of writing the anelastic momentum equation that has two great advantages over the primative form of that equation. First, it obviates the need to calculate the perturbation, $\mathbf{g}_{c}$, in $\mathbf{g}$ created by the perturbation, $\rho_{c}$, in density. If $\mathbf{g}_{c}$ is deemed to be interesting, it can be evaluated after the main part of the calculation has been completed. Second, it is clear on physical grounds that density variations produced by pressure variations, $p_{c}$, will not drive convection. The simplification allows that part of $\rho_{c}$ to be split off from $\rho_{c}$ and absorbed into the gradient term, so leaving the remaining variations in $\rho_{c}$ due to changes in entropy and composition to be included in the "co-density", $C$, which provides the buoyancy force. 


\section{Table 3: FULL CONVECTION EQUATIONS}

Write

$$
S=S_{a}+S_{c}, \quad \xi=\xi_{a}+\xi_{c}, \quad \ldots, \quad \mathbf{g}_{\mathrm{e}}=\mathrm{g}_{\mathrm{e} a}+\mathbf{g}_{c}, \quad U_{\mathrm{e}}=U_{\mathrm{e} a}+U_{c}
$$

where $S_{c}=\mathrm{O}\left(\epsilon_{c} S_{a}\right) \ldots$, but $\mathbf{V}_{a}=\mathrm{O}\left(\epsilon_{c} \mathbf{V}_{c}\right)$. Use two time scale methods:

$$
\partial_{t}=\partial_{t}^{a}+\partial_{t}^{c}
$$

where $\partial_{t}^{a}=\mathrm{O}\left(\epsilon_{c} \partial_{t}^{c}\right)$; also $d_{t}^{c}=\partial_{t}^{c}+\mathbf{V}_{c} \cdot \nabla$ and $\dot{f}=\partial_{t}^{a} f$.

Substitute in basic equations; retain only largest terms in $\epsilon_{c}$ :

$$
\begin{array}{lc}
\rho_{a} d_{t}^{c} \mathbf{V}_{c}=-\nabla p_{c}+\rho_{a} \mathbf{g}_{c}+\rho_{c} \mathbf{g}_{a}-2 \rho_{a} \Omega \times \mathbf{V}_{c}+\rho_{a} \mathbf{F}^{V}+\rho_{a} \mathbf{F}^{J}, & \nabla \cdot\left(\rho_{a} \mathbf{V}_{c}\right)=0 \\
\rho_{a} d_{t}^{c} \xi_{c}=-\nabla \cdot \mathbf{I}^{\xi}-\rho_{a} \dot{\xi}_{a}, & \rho_{a} d_{t}^{c} S_{c}=-\nabla \cdot \mathbf{I}^{S}-\rho_{a} \dot{S}_{a}+\sigma^{S} \\
\partial_{t} \mathbf{B}_{c}=\nabla \times\left(\mathbf{V}_{c} \times \mathbf{B}_{c}\right)-\nabla \times\left(\eta \nabla \times \mathbf{B}_{c}\right), & \nabla \cdot \mathbf{B}_{c}=0 \\
\mathbf{g}_{c}=-\nabla U_{c}, & \nabla^{2} U_{c}=4 \pi k_{N} \rho_{c}
\end{array}
$$

The second of these is the usual anelastic equation

A significant simplification:

The equation of motion can be re-written as

$$
d_{t}^{c} \mathbf{V}_{c}=-\nabla P_{c}+C_{c} \mathbf{g}_{a}-2 \Omega \times \mathbf{V}_{c}+\mathbf{F}^{V}+\mathbf{F}^{J}
$$

where

$$
C_{c}=-\alpha^{S} S_{c}-\alpha^{\xi} \xi_{c}=\text { codensity, } \quad P_{c}=p_{c} / \rho_{a}+U_{c}=\Pi_{c}-T_{a} S_{c}-\mu_{a} \xi_{c}
$$

Scale separation (step 4) is the subject of Table 4. Here the Reynolds stresses on the macroscale velocity are introduced. They give rise to the body forces $\mathbf{F}^{V^{\prime}}$ and $\mathbf{F}^{J^{\prime}}$. The viscous force associated with the mean motion is denoted by $\mathbf{F}^{\bar{\nu}}$. It appears to be negligible in the geophysical application except in boundary layers. The mean Lorentz force, $\mathbf{F}^{\bar{J}}$, is however very significant. The mean fluxes, $\mathbf{I}^{S^{\prime}}$ and $\mathbf{I}^{\xi^{\prime}}$, of entropy and composition created by the turbulence are also important.. It may be seen in Table 4 that the transport of heat "down the adiabat" of the reference state and the associated entropy production $\sigma^{T}$ are both included. These are not negligible in the Earth's core.

In preparation for step 5, the discussion of aspects of local turbulence theory (Table $5 \mathrm{c}$ ), Table 5a provides a reminder of the basic theory underlying the general expressions for entropy flux and compositional flux. This leads to a form for the associated entropy production, $\sigma^{m}$, and this has to be non-negative by the second law of thermodynamics. 


\section{Table 4: SCALE SEPARATION}

Write

$$
\mathbf{V}_{c}=\overline{\mathbf{V}}+\mathbf{V}^{\prime}, \quad \mathbf{B}_{c}=\overline{\mathbf{B}}+\mathbf{B}^{\prime}, \quad \mathbf{J}_{c}=\overline{\mathbf{J}}+\mathbf{J}^{\prime}, \ldots
$$

$\overline{\mathbf{V}}, \overline{\mathbf{B}}, \ldots=$ ensemble averages of $\mathbf{V}_{c}, \mathbf{B}_{c} \ldots, \mathbf{V}^{\prime}, \mathbf{B}^{\prime}, \ldots=$ turbulent parts of $\mathbf{V}_{c}, \mathbf{B}_{c} \ldots$

Average the basic convection equations:

\begin{tabular}{|ll|}
\hline$d_{t}^{\bar{c}} \overline{\mathbf{V}}=-\nabla \bar{P}+\bar{C} \mathbf{g}_{a}-2 \Omega \times \overline{\mathbf{V}}+\mathbf{F}^{\bar{\nu}}+\mathbf{F}^{\bar{J}}+\mathbf{F}^{V^{\prime}}+\mathbf{F}^{J^{\prime}}$, & $\nabla \cdot\left(\rho_{a} \overline{\mathbf{V}}\right)=0$, \\
$\rho_{a} d_{t}^{\bar{c}} \bar{\xi}=-\nabla \cdot \mathbf{I}^{\xi^{\prime}}-\rho_{a} \dot{\xi}_{a}, \quad \bar{C}=-\alpha^{S} \bar{S}-\alpha^{\xi} \bar{\xi}$ & \\
$\rho_{a} \bar{d}_{t}^{\bar{c}} \bar{S}=-\nabla \cdot \mathbf{I}^{S^{\prime}}+\nabla \cdot\left[\left(K^{T} / T_{a}\right) \nabla T_{a}\right]-\rho_{a} \dot{S}_{a}+\bar{\sigma}^{S}$ \\
$\partial_{t}^{c} \overline{\mathbf{B}}=\nabla \times(\overline{\mathbf{V}} \times \overline{\mathbf{B}})-\nabla \times(\eta \nabla \times \overline{\mathbf{B}}), \quad \nabla \cdot \overline{\mathbf{B}}=0$ \\
\hline
\end{tabular}

where $\quad d_{t}^{\bar{c}}=\partial_{t}^{c}+\overline{\mathbf{V}} \cdot \nabla, \quad \mathbf{I}^{\xi^{\prime}}=\rho_{a} \overline{\xi^{\prime} \mathbf{V}^{\prime}}, \quad \mathbf{I}^{S^{\prime}}=\rho_{a} \overline{S^{\prime} \mathbf{V}^{\prime}}, \quad \mathbf{F}^{\bar{J}}=\overline{\mathbf{J}} \times \overline{\mathbf{B}}$

$$
\begin{aligned}
& \rho_{a} \mathbf{F}^{V^{\prime}}=\nabla \cdot \overleftrightarrow{\pi}^{V^{\prime}}, \quad \overleftrightarrow{\pi}^{V^{\prime}}=-\rho_{a} \overline{\mathbf{V}^{\prime} \mathbf{V}^{\prime}}=\text { Reynolds stress } \\
& \rho_{a} \mathbf{F}^{J^{\prime}}=\nabla \cdot \overleftrightarrow{\pi} J^{\prime}, \quad \overleftrightarrow{\pi}^{J^{\prime}}=\mu_{0}^{-1} \overline{\mathbf{B}^{\prime} \mathbf{B}^{\prime}}=\text { Magnetic Reynolds stress } \\
& \bar{\sigma}^{S}=\sigma^{R}+\sigma^{T}+\sigma^{\bar{V}}+\sigma^{\bar{J}}+\sigma^{V^{\prime}}+\sigma^{J^{\prime}} \\
& \sigma^{T}=K^{T}\left(\nabla T_{a} / T_{a}\right)^{2}, \quad \sigma^{\bar{J}}=\eta \bar{J}^{2} / \mu_{0} T_{a}, \quad \sigma^{J^{\prime}}=\eta \overline{J^{\prime 2}} / \mu_{0} T_{a}, \ldots
\end{aligned}
$$

\section{Table 5a: TOTAL ENERGY BUDGET}

\section{Direct Consequences of Governing Equations:}

$$
\partial_{t} u^{\text {total }}+\nabla \cdot \mathbf{I}^{\text {total }}=T \sigma^{S}-Q^{V}-Q^{J}+\mathbf{I}^{S} \cdot \nabla T+\mathbf{I}^{\xi} \cdot \nabla \mu
$$

where

$u^{\text {total }}=\rho\left(\varepsilon^{I}+\varepsilon^{K}+U^{\Omega}\right)+u^{B}+u^{g}, \quad \mathbf{I}^{\text {total }}=\rho\left(\varepsilon^{H}+\varepsilon^{K}+U^{\Omega}\right) \mathbf{V}-\overleftrightarrow{\pi}^{V} \cdot \mathbf{V}+\mathbf{I}^{B}+\mathbf{I}^{g}+T \mathbf{I}^{S}+\mu \mathbf{I}$ $\varepsilon^{I}=$ internal energy $/ \mathrm{mass}, \quad \varepsilon^{H}=$ enthalpy $/$ mass, $\quad \varepsilon^{K}=$ kinetic energy $/ \mathrm{mass}$ $u^{B}=$ magnetic energy/volume,$\quad u^{g}=$ gravitational energy/volume,$\quad \varepsilon^{\Omega}=-\frac{1}{2}(\Omega \times \mathbf{x})^{2}$ $Q^{V}=$ viscous energy dissipated/volume $=T \sigma^{V}, Q^{J}=$ Joule energy dissipated $/$ volume $=T \sigma^{J}$ $\mathbf{I}^{B}=$ magnetic energy flux, $\quad \mathbf{I}^{g}=$ gravitational energy flux, $\quad \overleftrightarrow{\pi}^{V}=$ viscous stress tensor

What is required:

where

$$
\partial_{t} u^{\text {total }}+\nabla \cdot \mathbf{I}^{\text {total }}=Q^{R}
$$

$$
\begin{aligned}
& Q^{R}=\text { volumetric heat sources (radioactivity) } \\
& \mathbf{I}^{\text {total }}=\rho\left(\varepsilon^{H}+\varepsilon^{K}+U^{\Omega}\right) \mathbf{V}-\overleftrightarrow{\boldsymbol{\pi}}^{V} \cdot \mathbf{V}+\mathbf{I}^{B}+\mathbf{I}^{g}+\mathbf{I}^{q}, \quad \mathbf{I}^{q}=\text { heat flux vector }
\end{aligned}
$$

Important Results of Comparison:

$$
\begin{aligned}
\mathbf{I}^{q} & =T \mathbf{I}^{S}+\mu \mathbf{I}^{\xi} \\
\sigma^{S} & =\sigma^{R}+\sigma^{V}+\sigma^{J}-\left(\mathbf{I}^{S} \cdot \nabla T+\mathbf{I}^{\xi} \cdot \nabla \mu\right) / T
\end{aligned}
$$

Important Note: The second law of thermodynamics requires that

$$
\sigma^{m} \equiv-\left(\mathbf{I}^{S} \cdot \nabla T+\mathbf{I}^{\xi} \cdot \nabla \mu\right) / T \geq 0
$$


In Table $5 \mathrm{~b}$, we provide a reminder of how this is achieved when molecular transport is solely responsible for the fluxes of heat and composition. A more detailed account of this topic may be found in the text of Landau \& Lifshitz (1987). The corresponding local turbulence theory is given in Table 5c. This employs the Reynolds ansatz, and leads to an expression for the macroscale entropy production $\sigma^{t}$ created by the macroscale fluxes $\mathbf{I}^{S^{\prime}}$ and $\mathbf{I}^{\xi^{\prime}}$ generated by the turbulence. This expression is new and leads to values of $\sigma^{t}$ that, while not as large as some other sources of entropy, are significant in the application of the theory to GR2. The expression for $\sigma^{t}$ is negative in any domain where the reference state is stably stratified. That is not surprising since, according to local theory, turbulence cannot exist where the stratification is bottom heavy; penetration of turbulence from adjacent turbulent regions is excluded in a local theory. In such conditions therefore $\sigma^{t} \equiv 0$ and only the contribution, $\sigma^{m}$, made by molecular fluxes to entropy production is present. When $\sigma^{t}>0$ however, it normally greatly exceeds $\sigma^{m}$, and the neglect of the latter then results in only a very minor error.

\section{Table 5b: MOLECULAR DIFFUSION}

Question: Why is $\sigma^{m} \equiv-\left(\mathbf{I}^{S} \cdot \nabla T+\mathbf{I}^{\xi} \cdot \nabla \mu\right) / T \geq 0$ at the molecular level?

Answer: (cf. e.g., Landau \& Lifschitz, 1987)

Use $p, T$ and $\mu$ as independent variables and suppose that

$$
\mathbf{I}^{\xi}=-\alpha^{\prime} \nabla \mu-\beta^{\prime} \nabla T-\epsilon^{\prime} \nabla p, \quad \mathbf{I}^{S}=-\delta^{\prime} \nabla \mu-\gamma^{\prime} \nabla T-\zeta^{\prime} \nabla p
$$

We require that, for independent $\nabla \mu, \nabla T, \nabla p$,

$$
\begin{aligned}
& T \sigma^{m}=\alpha^{\prime}(\nabla \mu)^{2}+\gamma^{\prime}(\nabla T)^{2}+\left(\beta^{\prime}+\delta^{\prime}\right) \nabla \mu \cdot \nabla T+\epsilon^{\prime} \nabla p \cdot \nabla \mu+\zeta^{\prime} \nabla p \cdot \nabla T \geq 0 \\
& \Rightarrow \quad \alpha^{\prime} \geq 0, \quad \gamma^{\prime} \geq 0, \quad \epsilon^{\prime}=\zeta^{\prime}=0, \quad\left(\beta^{\prime}+\delta^{\prime}\right)^{2} \leq 4 \alpha^{\prime} \gamma^{\prime}
\end{aligned}
$$

Onsager reciprocity $\Rightarrow \quad \delta^{\prime}=\beta^{\prime}$.

Final result:

where

$$
\begin{array}{rlrl}
\mathbf{I}^{\xi} & =-\alpha^{\prime} \nabla \mu-\beta^{\prime} \nabla T, & \mathbf{I}^{S}=-\beta^{\prime} \nabla \mu-\gamma^{\prime} \nabla T \\
T \sigma^{m} & =\alpha^{\prime}(\nabla \mu)^{2}+\gamma^{\prime}(\nabla T)^{2}+2 \beta^{\prime} \nabla \mu \cdot \nabla T & \geq 0 \\
\alpha^{\prime} & \geq 0 & \alpha^{\prime} \gamma^{\prime}-\beta^{\prime 2} & \geq 0
\end{array}
$$

Note: Relation to the usual transport coefficients:

$\alpha^{\prime} \propto$ compositional diffusivity $=\kappa^{\xi} \geq 0$

$\alpha^{\prime} \gamma^{\prime}-\beta^{\prime 2} \propto$ thermal conductivity $=K^{T} \geq 0$

$\beta^{\prime}$ is related to the Soret coefficient (either sign)

$$
s^{m}=\sigma^{T}+\sigma^{\xi}, \quad \sigma^{T}=K^{T}\left[\frac{\nabla T}{T}\right]^{2} \geq 0, \quad \sigma^{\xi}=\frac{\mu_{T}^{\xi}}{\rho \kappa^{\xi} T}\left(\mathbf{I}^{\xi}\right)^{2} \geq 0
$$

where $\mu_{T}^{\xi}=(\partial \mu / \partial \xi)_{p, T}$ ( $\geq 0$ by thermodynamics $)$. 


\section{Table 5c: LOCAL TURBULENCE THEORY}

The Reynolds Ansatz: Treat eddies like molecules! The macroscopic fluxes of entropy and composition, and the associated entropy production, created by the turbulence are

$$
\begin{array}{ll}
\mathbf{I}^{S^{\prime}} \equiv \rho_{a} \overline{S^{\prime} \mathbf{V}^{\prime}}=-\rho_{a} \overleftrightarrow{\kappa}^{t} \cdot \nabla \bar{S}, & \mathbf{I}^{\xi^{\prime}} \equiv \rho_{a} \overline{\bar{\xi}^{\prime} \mathbf{V}^{\prime}}=-\rho_{a} \overleftrightarrow{\kappa}^{t} \cdot \nabla \bar{\xi} \\
\bar{\sigma}^{S}=\sigma^{R}+\sigma^{T}+\sigma^{\bar{V}}+\sigma^{\bar{J}}+\sigma^{m}+\sigma^{t}, & T_{a} \sigma^{t}=-\mathbf{I}^{S^{\prime}} \cdot \nabla\left(T_{a}+\bar{T}\right)-\mathbf{I}^{\xi^{\prime}} \cdot \nabla\left(\mu_{a}+\bar{\mu}\right)
\end{array}
$$

where $\overleftrightarrow{\kappa}^{t}=$ turbulent diffusivity tensor, which requires a separate analysis; see Braginsky \& Meytlis (1990) and Appendix C of BR. We should now ask three questions:

Question 1: Why is $\sigma^{t} \geq 0$ ?

Answer: Since $\epsilon_{c} \ll 1$, we have

Substitute gradients for reference state:

$$
T_{a} \sigma^{t} \approx-\mathbf{I}^{S^{\prime}} \cdot \nabla T_{a}-\mathbf{I}^{\xi^{\prime}} \cdot \nabla \mu_{a}
$$

where

$$
T_{a} \sigma^{t}=-\mathbf{I}^{S^{\prime}} \cdot\left(\alpha^{S} \mathbf{g}_{a}\right)-\mathbf{I}^{\xi^{\prime}} \cdot\left(\alpha^{\xi} \mathbf{g}_{a}\right)=\mathrm{g}_{a} \cdot \mathbf{I}^{C^{\prime}}
$$

$$
\mathbf{I}^{C^{\prime}}=-\alpha^{S} \mathbf{I}^{S^{\prime}}-\alpha^{\xi} \mathbf{I}^{\xi^{\prime}}=-\rho_{a} \overline{\left(\alpha^{S} S^{\prime}+\alpha^{\xi} \xi^{\prime}\right) \mathbf{V}^{\prime}}=\rho_{a} \overline{C^{\prime} \mathbf{V}^{\prime}}
$$

by definition. Thus $T_{a} \sigma^{t}=\rho_{a} \overline{C^{\prime} \mathbf{g}_{a} \cdot \mathrm{V}^{\prime}} \Rightarrow$

1. if $\sigma^{t}>0$, turbulence feeds on gravitational energy release in locally unstable stratification. (Usually $\sigma^{t} \gg \sigma^{m}$, so that $\sigma^{m}$ can be neglected.)

2. if $\sigma^{t}<0$, the stratification is locally bottom heavy; no energy available to drive turbulence. Therefore no local turbulence, and $\sigma^{t} \equiv 0$. (Small $\sigma^{m}>0$ still present)

Question 2: How can $\sigma^{t}$ be calculated?

Answer: We have

$$
T_{a} \sigma^{t}=\mathbf{g}_{a} \cdot \mathbf{I}^{C^{\prime}}=\rho_{a} \mathbf{g}_{a} \cdot \overleftrightarrow{\kappa}^{t} \cdot\left(\alpha^{S} \nabla \bar{S}+\alpha^{\xi} \nabla \bar{\xi}\right)
$$

Question 3: Is $\sigma^{t}=\sigma^{V^{\prime}}+\sigma^{J^{\prime}}$, as required for consistency?

Answer: Yes. It is a consequence of assuming that either microscale magnetic Reynolds number $v \ell / \eta$ is small or that the microscale kinetic Reynolds number $v \ell / \nu$ is small, or both, where $v$ and $\ell$ are velocity and length scales characteristic of the turbulence. See BR

The equations governing the reduced anelastic theory (step 6A) are summarized in Table 6. Solutions are required to satisfy a number of boundary conditions, the most awkward of which arise at the ICB. They require knowledge of the properties of the liquidus sketched in Figure 2, and govern the release of latent heat and light constituent; they therefore determine the fluxes $\mathbf{I}^{S^{\prime}}$ and $\mathbf{I}^{\xi^{\prime}}$ at the ICB. They raise complicated issues, and the numerical values of the pertinent coefficients are very uncertain. The topic is discussed both in Appendix E of BR and in the formulation of GR2 but not here. Among the other boundary conditions that must be satisfied are the no-slip conditions at the CMB and ICB. The SIC is allowed to turn about the geographical axis, which is parallel to $\Omega$, in response to the viscous and electromagnetic torques to which the FOC subjects it. It is assumed to be electrically conducting, its magnetic diffusivity being the same as that of the FOC. The magnetic field is required to be continuous across the ICB and the FOC. The mantle is assumed to be conducting in a thin layer at its base. The flux of mass into the CMB is supposed to be zero, so that $\mathbf{I}^{\xi^{\prime}}=0$ there. The flux $T_{a} \mathbf{I}^{S^{\prime}}$ is the flux of heat from core to mantle, and must be specified. 
Table 6: FINAL CONVECTION EQUATIONS

Simplify Notation: $\overline{\mathbf{V}} \rightarrow \mathrm{V}, \bar{S} \rightarrow S, \ldots, d_{t}^{\bar{c}} \rightarrow d_{t}$

Simplify Physics: Ignore $\mathrm{F}^{V^{\prime}}$ and $\mathrm{F}^{J^{\prime}}$; probably insignificant in geophysical context;

$\mathrm{F}^{V}$ important only in boundary layers

Simplified Theory:

$$
\begin{aligned}
& d_{t} \mathbf{V}=-\nabla P+C \mathbf{g}_{a}-2 \Omega \times \mathbf{V}+\mathbf{F}^{V}+\mathbf{F}^{J} \\
& \nabla \cdot\left(\rho_{a} \mathbf{V}\right)=0, \quad C=-\alpha^{S} S-\alpha^{\xi} \xi \\
& \rho_{a} d_{t} \xi=-\nabla \cdot \mathbf{I}^{\xi^{\prime}}-\rho_{a} \dot{\xi}_{a} \\
& \rho_{a} d_{t} S=-\nabla \cdot \mathbf{I}^{S^{\prime}}+T_{a}^{-1} \nabla \cdot\left[K^{T} \nabla T_{a}\right]-\rho_{a} \dot{S}_{a}+\sigma^{S} \\
& \partial_{t} \mathbf{B}=\nabla \times(\mathbf{V} \times \mathbf{B})-\nabla \times(\eta \nabla \times \mathbf{B}), \quad \nabla \cdot \mathbf{B}=0
\end{aligned}
$$

where

$$
\begin{array}{ll}
\mathbf{I}^{S^{\prime}}=-\rho_{a} \overleftrightarrow{\kappa}^{t} \cdot \nabla S, & \mathbf{I}^{\xi^{\prime}}=-\rho_{a} \overleftrightarrow{\kappa}^{t} \cdot \nabla \xi \\
\sigma^{S}=\sigma^{R}+\sigma^{T}+\sigma^{V}+\sigma^{J}+\sigma^{m}+\sigma^{t}, & \sigma^{t}=\rho_{a} \mathbf{g}_{a} \cdot \overleftrightarrow{\kappa}^{t} \cdot\left(\alpha^{S} \nabla S+\alpha^{\xi} \nabla \xi\right) / T_{a}
\end{array}
$$

Further simplifications customarily adopted:

1. Ignore effect of centrifugal forces on reference state:

$$
\epsilon_{\Omega} \rightarrow 0
$$

Then reference state is spherically symmetric

2. Assume turbulence is isotropic:

$$
\kappa_{i j}^{t}=\kappa \delta_{i j}
$$

\section{The Strategy in Application}

As mentioned in $\S 1$, the anelastic theory has recently been applied to a simulation of the geodynamo (GR2), driven by cooling of 7.2TW uniformly distributed over the CMB. Since the results will soon appear in print, we shall confine ourselves here to a few of the more interesting findings. First, the magnetic field was maintained for 160,000 yrs of simulated time, which is an order of magnitude greater than the free decay time, $R_{1}^{2} / \pi^{2} \eta$, in which the field would disappear through Joule losses in the absence of electric currents induced by the fluid motion. There seems little doubt that dynamo action will maintain the field for as long as computer resources permit the numerical integrations to be continued. Second, and encouragingly, the simulated field resembles the observed geomagnetic field in a number of ways. It is of about the right strength and is dipole-dominated. Its power spectrum is slightly red at the CMB. The field patterns drift predominantly westward, at about the same angular speed as do those of the real geomagnetic field.

The forms of the fields $S$ and $\xi$ are closely similar everywhere in the FOC except near the CMB, where the different boundary conditions they satisfy make them behave differently. Their similarity elsewhere is a consequence of the fact that the source of one is the latent heat release at the ICB and of the other is the light constituent release at the ICB. These sources vary in strength over the ICB but are always proportional to the 
the local rate of freezing and therefore to one another. Thus the $S$ and $\xi$ they produce are also proportional. This adds support to previous investigations that assumed that compositional buoyancy could be incorporated into thermal buoyancy in core modeling.

As for the earlier models GR1 \& GR2, the inner core rotates predominantly eastward at between $1^{\circ} / \mathrm{yr}$ and $2^{\circ} / \mathrm{yr}$. This prediction, made by Glatzmaier \& Roberts (1995a), was taken seriously by two groups of seismologists, Song \& Richards (1996) and Su et al. (1996), who by analysis of past seismic records claim to detect a rotation of Earth's inner core in the eastward direction and of approximately the magnitude we suggested.

The most exciting aspect of model GR1 was that, during the period between $36,000 \mathrm{yr}$ and 40,000 yrs after its initiation, the field reversed its polarity spontaneously, in much the same way as the observed geomagnetic field has done many times in its history (Glatzmaier \& Roberts, 1995b).

After numerous reversals, the future of geodynamo theory seems bright!

\section{Acknowledgments}

I have benefitted from conversations with Drs Stanislav Braginsky and Gary Glatzmaier about the subject expounded above. This work was supported in part by the Yamada foundation and in part by the National Science Foundation under grant EAR94-06002.

\section{References}

Braginsky, S.I., "Structure of the F layer and reasons for convection in the Earth's core," Soviet Phys. Dokl., 149, 8-10 (1963).

Braginsky, S.I. \& Meytlis, V.P., "Local turbulence in the Earth's core," Geophys. Astrophys. Fluid Dynam., 55, 71 - 87 (1990).

Braginsky, S.I. \& Roberts, P.H., "Equations governing convection in Earth's core and the Geodynamo," Geophys. Astrophys. Fluid Dynam., 79, 1 - 97 (1995).

Dziewonski, A.M. \& Anderson, D.L., "Preliminary reference Earth model," Phys. Earth Planet. Inter., 25, 297 - 356 (1981).

Glatzmaier, G.A. \& Roberts, P.H., "A three-dimensional convective dynamo solution with rotating and finitely conducting inner core and mantle", Phys. Earth Planet. Inter., 91, 63 - 75 (1995a).

Glatzmaier, G.A. \& Roberts, P.H., "A simulated geomagnetic reversal", Nature, 377, 203 -208 (1995b).

Glatzmaier, G.A. \& Roberts, P.H., "On the magnetic sounding of planetary interiors", Phys. Earth Planet. Inter., to appear (1996a).

Glatzmaier, G.A. \& Roberts, P.H., "An anelastic evolutionary geodynamo simulation driven by composition and thermal convection", Physica D, to appear (1996b).

Kageyama, A., Sato, T., Watanabe, K., Horiuchi, R., Hayashi, T., Todo, Y., Watanabe, T.H. \& Takamara, H., "Computer simulation of a magnetohydrodynamic dynamo II", Phys. Plasmas, 2, 1421 - 1431 (1995).

Krause, F. \& Rädler, K.-H., Mean Field Magneto-Hydrodynamics and Dynamo Theory, Oxford: Pergamon (1980).

Landau, L.D. \& Lifshitz, E.M., Fluid Mechanics, $2^{\text {nd }}$ Edition. Oxford: Pergamon (1987).

Song, X.D. \& Richards, P.G., "Rotation of Earth's inner core", Nature, to appear (1996).

$\mathrm{Su}$, W.-J, Dziewonski, A.M. \& Jeanloz, R., "Planet within a planet: Rotation of the inner core of the Earth", Submitted to Science, (1996). 